\title{
Disseminated Gastrointestinal Mucormycosis in Immunocompromised Disease
}

\author{
Tae Sun Ha, M.D. ${ }^{1}$, Chi-Min Park, M.D. ${ }^{1}$, Jeong Hoon Yang, M.D. ${ }^{1,2}$, Yang Hyun Cho, M.D. ${ }^{1,3}$, \\ Chi Ryang Chung, M.D. ${ }^{1}$, Kyeongman Jeon, M.D. ${ }^{1,4}$, and Gee Young Suh, M.D. ${ }^{1,4}$ \\ ${ }^{1}$ Department of Critical Care Medicine, Department of Surgery, ${ }^{2}$ Division of Cardiology, Department of Medicine, ${ }^{3}$ Department of Thoracic and Cardiovascular Surgery, ${ }^{4}$ Division of \\ Pulmonary and Critical Care Medicine, Department of Medicine, Samsung Medical Center, Sungkyunkwan University School of Medicine, Seoul, Korea
}

Mucormycosis is an uncommon opportunistic fungal infection mostly affecting immunocompromised patients and gastrointestinal mucormycosis is a rare and life-threatening. We describe a 31-year-old man with a history of idiopathic cyclic neutropenia who developed perforations of the stomach and intestine and intra-abdominal bleeding due to disseminated gastrointestinal mucormycosis after the initial operation.

Key Words: gastrointestinal tract; immunocompromised host; mucormycosis.

Mucormycosis, classified within the Mucorales order of the zygomycetes class, is an opportunistic omnipresent fungus found in soil and decaying organic matter. Human mucormycosis infections, caused by either inhalation or ingestion of fungal sporangiospores, are extremely rare but result in high mortality. Due to an increasing prevalence of patients with predisposing factors for mucormycosis such as hematological malignancies, post-transplantation, poorly controlled diabetes mellitus, prolonged use of corticosteroid and severe malnutrition, the incidence and importance of Mucorales have increased over the last few decades.[1] Mucormycosis is classified as rhinocerebral, pulmonary, cutaneous, gastrointestinal, central nervous system, disseminated or miscellaneous based on the clinical presentation and the anatomical involvement. Of these, gastrointestinal (GI) mucormycosis is an especially rare and life-threatening disease in immunocompromised patients.[2] We report a case of a fatal disseminated gastrointestinal mucormycosis in a patient with immunocompromised disease.

\section{Case Report}

A 31-year-old male patient with a history of idiopathic cyclic neutropenia and biopsy-proven non-definite abnormalities presented to the emergency room (ER) with severe abdominal pain, fever and watery diarrhea. The patient had been prescribed tacrolimus and cyclophosphomide for idiopathic neutropenia

Received on April 7, 2015 Revised on May 27, 2015

Accepted on June 24, 2015

Correspondence to: Chi-Min Park, Department of Critical Care Medicine, Department of Surgery, Samsung Medical Center, Sungkyunkwan University

School of Medicine, 81 Irwon-ro, Gangnam-gu, Seoul 06351, Korea

Tel: +82-2-3410-1096, Fax: +82-2-2148-7088

E-mail: dr99.park@samsung.com

*No potential conflict of interest relevant to this article was reported. for two months starting in April 2014 and had not used H2blockers, proton pump inhibitors, corticosteroids or broadspectrum antibiotics in the previous six months. On arrival in the ER, he was alert and well-oriented, with a blood pressure of $135 / 95 \mathrm{mmHg}$, a pulse rate of $124 / \mathrm{min}$, a body temperature of $37.3^{\circ} \mathrm{C}$, and an oxygen saturation of $99 \%$ on room air. Abdominal examination revealed a soft, distended, and tender 
right lower quadrant and rebound tenderness with sluggish bowel sounds. The laboratory data on arrival were as follows: white blood cells $260 / \mu \mathrm{L}$, absolute neutrophil count 3 $/ \mu \mathrm{L}$, PH 7.311, HCO3 $13.7 \mathrm{mmol} / \mathrm{L}$, creatinine $1.85 \mathrm{mg} / \mathrm{dL}$, total bilirubin $6.6 \mathrm{mg} / \mathrm{dL}$, aspartate aminotransferase/alanine aminotransferase 150/94 IU/L, C-reactive protein $26.22 \mathrm{mg} /$ $\mathrm{dL}$ and lactate $9.32 \mu \mathrm{mol} / \mathrm{L}$. The coagulation profile was normal. Hepatitis B, C and human immunodeficiency virus serology were negative, and three sets of blood cultures

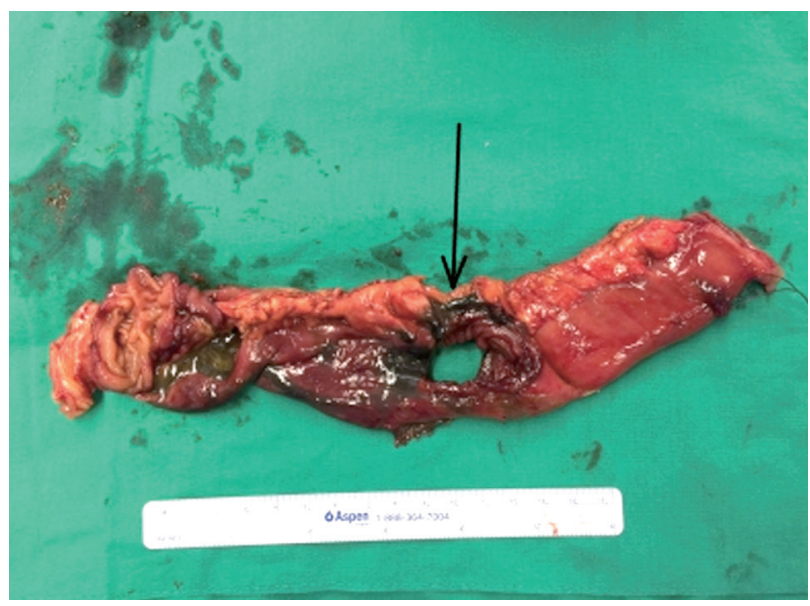

Fig. 1. Black ulcer with full bowel infarction of the small intestine (arrow) on the exposed specimen taken during the second operation. were also negative. Abdominal computed tomography (CT) with contrast showed acute typhlitis involving the terminal ileum, cecum, and proximal ascending colon with focal perforation. An emergency laparotomy revealed a large ascites collection and focal gangrene of the proximal ascending colon without perforation, and a right hemicolectomy was performed. Histopathological exam showed hemorrhagic colon infarction without an identified pathogen. Postoperatively, the patient developed severe neutropenia and overwhelming septic shock. He received early goal-directed therapy with use of broad spectrum antibiotics (vancomycin, meropenem), high-dose vasopressors (norepinephrine $1.0 \mathrm{mg} / \mathrm{min} /$ $\mathrm{kg}$, vasopressin $0.03 \mathrm{mg} / \mathrm{min} / \mathrm{kg}$ ), and cytokine therapy with granulocyte colony-stimulating factor (G-CSF) and granulocyte-macrophage colony-stimulating factor (GM-CSF) due to severe neutropenia, and hemoperfusion was conducted with two sets of polymyxin B immobilized fiber columns (Toraymyxin ${ }^{\circledR}$ PMX-F; Toray Industries, Tokyo, Japan). The patient gradually stabilized and recovered well. Two weeks later, he abruptly developed a high fever and severe abdominal pain with bile drainage from a Jackson-Pratt (JP) drain. Abdominal CT showed a large pneumoperitoneum and perforation of the proximal jejunum. An exploratory laparotomy was performed using small bowel segmental re-
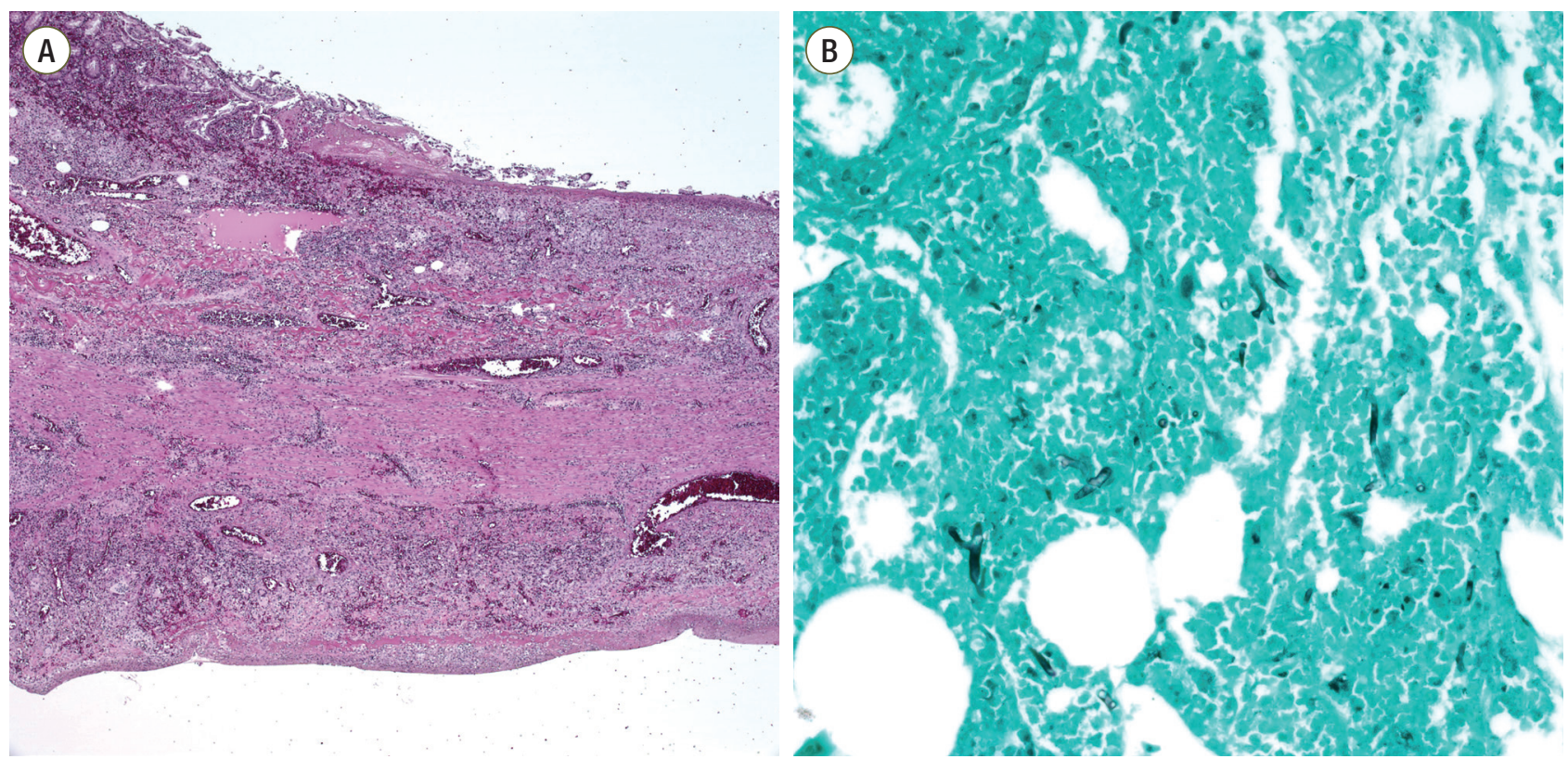

Fig. 2. Microscopic findings of the resected small bowel. (A) Microscopic findings of the small bowel showed vasculitis and transmural infarction (staining, H\&E; magnification, x40), (B) GMS staining revealed broad, irregular and non-septate fungal hyphae (staining, GMS; magnification. x200). H\&E: Hematoxylin and eosin; GMS: Gomori's methenamine-silver stain. 


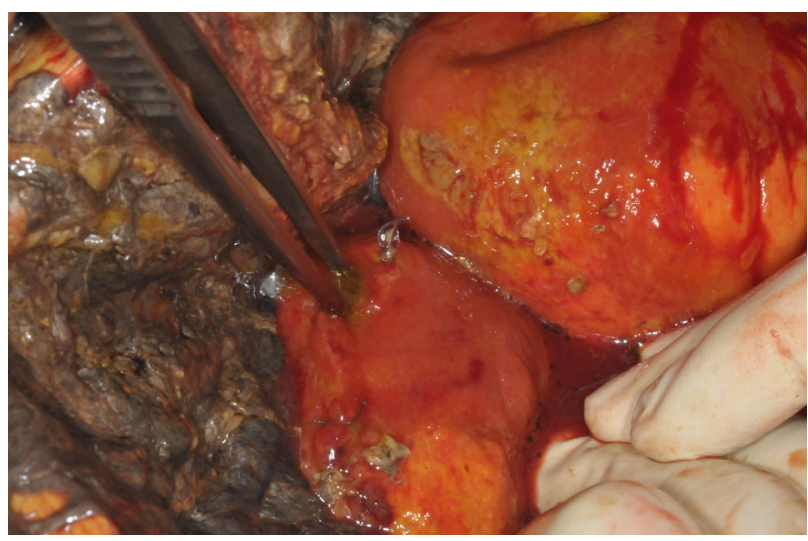

Fig. 3. Necrosis of the omentum and perforation of the transverse colon.

section and anastomosis (Fig. 1). Histopathological exam identified fungal hyphae as invasive mucormycosis with vasculitis and segmental transmural infarction with perforation (Fig. 2). He was started on the antifungal agent liposomal amphotericin B ( $5 \mathrm{mg} / \mathrm{kg} /$ day) for gastrointestinal mucormycosis. In the course of his slow recovery, he presented with abdominal distension and bowel content drainage via the JP drain on day 25 after the first operation. Abdominal CT showed a larger pneumoperitoneum and ascites. An emergency laparotomy was again performed, and necrosis of the omentum and a $5-\mathrm{cm}$ and $1-\mathrm{cm}$ perforation with indurated edges were identified over the stomach and transverse colon, respectively (Fig. 3). Primary repair of the stomach and transverse colon and an omentectomy were performed. Mucormycosis was confirmed on histological examination of the stomach and transverse colon, as expected. After the third laparotomy, four more exploratory laparotomies were performed due to the development of multiple separate perforations of the small bowel and intermittent intra-abdominal bleeding in the mesentery of the small bowel or peritoneum due to disseminated infection (Fig. 4). In conclusion, mucormycosis involved the stomach, small bowel, colon, omentum, and peritoneum, but the other sites, including sinuses, lungs, skin, and brain, have not been manifested. In spite of extensive surgical debridement and maximal medical support, the patient's condition deteriorated, and he died 49 days after the first laparotomy.

\section{Discussion}

Mucorales are saprobiotic organisms ubiquitous in nature

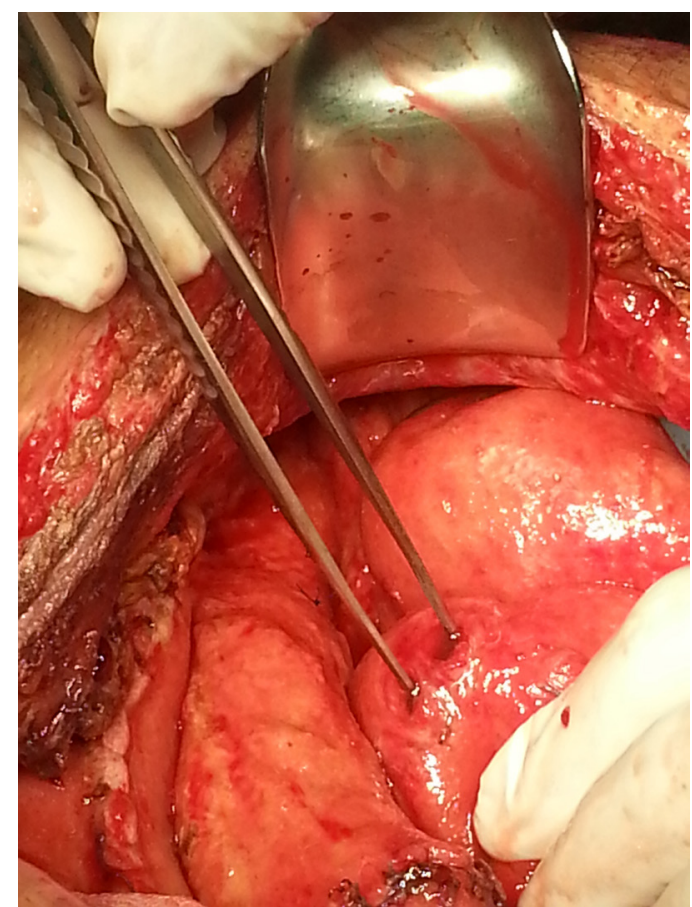

Fig. 4. Multiple perforations of the small bowel discovered during the sixth operation.

and opportunistic pathogens in humans. The majority of human infections arise from inhalation of fungal sporangiospores that have been released in the air or by direct inoculation of organisms into disrupted skin or mucosa.[3] The organism grows rapidly and produces hyaline aseptate hyphae in tissues, which then invade and disseminate.[4] The most common species of the Mucoraceae family confirmed in human disease are the genera Rhizopus, Lichtheimia, and Mucor.[4,5] Owing to the low virulence of these organisms, infections are seen within a variety of disease states that cause immunosuppression, including malignant hematological disease with or without stem cell transplantation, immunosuppressive therapy after transplantation, prolonged use of corticosteroids, prolonged and severe neutropenia, poorly controlled diabetes mellitus with or without diabetic ketoacidosis, use of antifungal agents with no activity against mucormycosis such as voriconazole or caspofungin, neonatal prematurity and severe malnutrition.[1,6] Mucormycosis is classified as rhinocerebral, pulmonary, cutaneous, gastrointestinal, central nervous system, disseminated or miscellaneous based on the clinical presentation and anatomical site involved. The common reported sites of mucormycosis are the sinuses (39\%), lungs (24\%), skin (19\%), brain (9\%), 
gastrointestinal tract (7\%), and disseminated infection (3\%). The mortality rate of mucormycosis has been reported as $96 \%$ for disseminated infections, $85 \%$ for gastrointestinal infections, and $76 \%$ for pulmonary infections.[1,5,6]

The aforementioned gastrointestinal mucormycosis is rare and is thought to arise from ingestion of the fungi in food or contaminated sputum. It can develop in any part of the alimentary tract, but the most commonly involved site is the stomach $(57.5 \%)$, followed by the colon $(32.3 \%)$, ileum $(7 \%)$, esophagus $(7 \%)$, duodenum $(2.3 \%)$, and jejunum (1.1\%).[7] The clinical hallmark of mucormycosis infection is tissue infarction and necrosis due to angioinvasion and subsequent thrombosis and a strong propensity for hematogenous dissemination. The symptoms vary depending on the involved site, but initial presentations are generally nonspecific abdominal pain and distension, fever, and diarrhea. If the bowel walls and blood vessels are involved with multiple ulcers caused by the fungal infection, this may result in bowel hemorrhage and perforation, peritonitis and sepsis, which is the most common cause of death.[8] In Korea, several case reports regarding GI mucormycosis which were developed in patients with immunodeficiency, including chronic renal failure, kidney transplantation, hematologic malignancy, and alcoholic liver cirrhosis, were published.[912] There are only a few reports of successfully treated invasive GI mucormycosis due to its high mortality rate.[9-11] As the majority of physicians are not very familiar with the disease, diagnosis is usually delayed. Thus, persistent severe abdominal pain in patients with neutropenia should alert clinicians to the possibility of invasive mucormycosis. When the clinical presentation suggests mucormycosis, there are no reliable serologic or skin tests. CT and magnetic resonance imaging are helpful for the diagnosis, as they can detect a decreased attenuation of the bowel wall resulting from edema, infarction or necrosis and a pneumoperitoneum or pneumatosis intestinalis associated with perforation or ischemia. Histopathological examination for fungal invasion of a tissue biopsy after surgery or endoscopy of the suspected area confirms the clinical or radiological diagnosis.

In our patient with idiopathic neutropenia, an emergency laparotomy was performed to identify the perforation of the proximal ascending colon during the first operation. However, only a dilated cecum and ascending colon with an edematous wall and color change due to hemorrhagic infarction were noted, but no perforation was found and no characteristic hyphae in the sections of tissue obtained were observed on histopathological examination. We considered that the ischemic damage resulted from septic shock and therefore performed aggressive medical therapy with broad-spectrum antibiotics for septic shock in the intensive care unit. When the second operation revealed a perforation of the proximal jejunum, the cause was eventually revealed to be invasive gastrointestinal mucormycosis. Despite antifungal therapy, disseminated GI mucormycosis had progressed and invaded multiple mesentery vessels, which caused uncontrolled fatal intra-abdominal bleeding.

The following are fundamental in the management of gastrointestinal mucormycosis: prompt diagnosis, correction of the underlying predisposing factors, aggressive surgical debridement of involved tissues and rapid initiation of antifungal therapy. GI mucormycosis is frequently rapidly progressive and has a high propensity for angioinvasion, resulting in thrombosis and tissue necrosis. As a result, antifungal agents show poor penetration to the site of infection. Therefore, early diagnosis and prompt initiation of treatment are essential for a favorable survival. Roden et al.[6] showed that surgical treatment combined with antifungal therapy improves the outcome compared to antifungal therapy alone or surgery alone. Early surgical debridement of infected or necrotic tissue as possible is role of surgery for survival benefit.[13] Currently, the recommended antifungal therapy includes amphotericin B and its lipid derivatives, which are active against mucormycosis and have a broad range of susceptibilities for various species that cause mucormycosis. [14] The doses of amphotericin B and amphotericin lipid derivatives with less nephrotoxicity are 1 to $1.5 \mathrm{mg} / \mathrm{kg} /$ day and $5 \mathrm{mg} / \mathrm{kg} /$ day, respectively.[15] Other antifungal agents, including itraconazole, voriconazole and caspofugin, have shown no or minimal activity against the Mucorales in in vitro studies or in clinic.[16] However, posaconazole, a novel broad-spectrum oral triazole, seems to possess activity against the Mucorales and may be considered as salvage therapy for refractory mycosis.[17] Apart from combined surgical and medical therapy, correction of the underlying predisposing factors such as withdrawal of corticosteroids, tapering of immunosuppression medications if possible and correction of metabolic abnormalities should be performed. Recovery from neutropenia in patients with mucormycosis 
is correlated with favorable outcome; thus, G-CSF and GMCSF are routinely administered to reduce the degree and duration of neutropenia.[18,19] In our patent, the diagnosis of mucormycosis was confirmed after the second operation, at which time we began antifungal therapy with liposomal amphotericin B and cytokine therapy with G-CSF and GMCSF. We consider use of posaconazole as salvage therapy for disseminated GI mucormycosis. However, posaconazole have high efficiency if the drug was administered with a meal, and posaconazole bioavailability is vulnerable to gastrointestinal disorder and food intake.[20] Owing to the repeat of gastrointestinal perforation and bleeding, combination of posaconazole with liposomal amphotericin B could not be used in our patient of nil per os status.

In conclusion, we report a case of disseminated gastrointestinal mucormycosis in an immunocompromised patient. Mucormycosis is a rare but emerging fungal infection with a high mortality rate. Commonly, antifungal therapy is applied only when histological examination of mucormycosis is confirmed several days following surgery or endoscopy, and it is too late to alter the clinical course of the fatal disease. Therefore, based on a high index of suspicion for mucormycosis in patients with predisposing factors, we consider that timely diagnosis, aggressive surgical debridement of infected tissue, and early initiation of amphotericin B therapy are essential for successful treatment.

\section{References}

1) Petrikkos G, Skiada A, Lortholary O, Roilides E, Walsh TJ, Kontoyiannis DP: Epidemiology and clinical manifestations of mucormycosis. Clin Infect Dis 2012; 54 Suppl 1: S23-34.

2) Gonzalez CE, Rinaldi MG, Sugar AM: Zygomycosis. Infect Dis Clin North Am 2002; 16: 895-914, vi.

3) Petrikkos G, Drogari-Apiranthitou M: Zygomycosis in Immunocompromised non-Haematological Patients. Mediterr J Hematol Infect Dis 2011; 3: e2011012.

4) Prabhu RM, Patel R: Mucormycosis and entomophthoramycosis: a review of the clinical manifestations, diagnosis and treatment. Clin Microbiol Infect 2004; 10 Suppl 1: 31-47.

5) Ribes JA, Vanover-Sams CL, Baker DJ: Zygomycetes in human disease. Clin Microbiol Rev 2000; 13: 236301.

6) Roden MM, Zaoutis TE, Buchanan WL, Knudsen TA, Sarkisova TA, Schaufele RL, et al: Epidemiology and outcome of zygomycosis: a review of 929 reported cases. Clin Infect Dis 2005; 41: 634-53.

7) Agha FP, Lee HH, Boland CR, Bradley SF: Mucormycoma of the colon: early diagnosis and successful management. AJR Am J Roentgenol 1985; 145: 739-41.

8) Lo OS, Law WL: Ileocolonic mucormycosis in adult immunocompromised patients: a surgeon's perspective. World J Gastroenterol 2010; 16: 1165-70.

9) Lee SH, Son YG, Sohn SS, Ryu SW: Successful treatment of invasive gastric mucormycosis in a patient with alcoholic liver cirrhosis: a case report. Exp Ther Med 2014; 8: 401-4.

10) Yang YC, Park CK, Kim JS, Park NH, Lee SH, Kim $\mathrm{SR}$, et al: A case of mucormycosis colonization associated with giant gastric ulcer. Korean J Gastrointest Endosc 2000; 20: 49-52.

11) Han JY, Cheon JH, Kim DH, Chon HJ, Kim SK, Kim TI, et al: Ileal mucormycosis diagnosed by colonoscopy in a patient with acute myeloid leukemia. Korean J Gastroenterol 2008; 52: 179-82.

12) Suh IW, Park CS, Lee MS, Lee JH, Chang MS, Woo $\mathrm{JH}$, et al: Hepatic and small bowel mucormycosis after chemotherapy in a patient with acute lymphocytic leukemia. J Korean Med Sci 2000; 15: 351-4.

13) Kontoyiannis DP, Wessel VC, Bodey GP, Rolston KV: Zygomycosis in the 1990s in a tertiary-care cancer center. Clin Infect Dis 2000; 30: 851-6.

14) Kontoyiannis DP, Lewis RE: Invasive zygomycosis: update on pathogenesis, clinical manifestations, and management. Infect Dis Clin North Am 2006; 20: 581607, vi.

15) Spellberg B, Walsh TJ, Kontoyiannis DP, Edwards J Jr, Ibrahim AS: Recent advances in the management of mucormycosis: from bench to bedside. Clin Infect Dis 2009; 48: 1743-51.

16) Gómez-López A, Cuenca-Estrella M, Monzón A, Rodriguez-Tudela JL: In vitro susceptibility of clinical isolates of Zygomycota to amphotericin B, flucytosine, itraconazole and voriconazole. J Antimicrob Chemother 2001; 48: 919-21. 
17) van Burik JA, Hare RS, Solomon HF, Corrado ML, Kontoyiannis DP: Posaconazole is effective as salvage therapy in zygomycosis: a retrospective summary of 91 cases. Clin Infect Dis 2006; 42: e61-5.

18) Liles WC, Huang JE, van Burik JA, Bowden RA, Dale DC: Granulocyte colony-stimulating factor administered in vivo augments neutrophil-mediated activity against opportunistic fungal pathogens. J Infect Dis 1997; 175: 1012-5.
19) Garcia-Diaz JB, Palau L, Pankey GA: Resolution of rhinocerebral zygomycosis associated with adjuvant administration of granulocyte-macrophage colonystimulating factor. Clin Infect Dis 2001; 32: e145-50.

20) Vaes M, Hites M, Cotton F, Bourguignon AM, Csergö $\mathrm{M}$, Rasson $\mathrm{C}$, et al: Therapeutic drug monitoring of posaconazole in patients with acute myeloid leukemia or myelodysplastic syndrome. Antimicrob Agents Chemother 2012; 56: 6298-303. 